\title{
Las TIC y las estrategias de enseñanza aplicadas en el desarrollo de las clases virtuales en las materias de química y física en el nivel medio del colegio nacional San Javier en el año 2020-2021
}

\author{
Luisa Concepción Escurra Álvarez \\ luisaescurra@hotmail.com \\ Facultad de Humanidades y Ciencias de la Educación \\ Universidad Nacional de Pilar \\ Pilar - Paraguay
}

\section{RESUMEN}

La investigación denominada Las TIC y las estrategias de enseñanza aplicadas en el desarrollo de las clases virtuales en las materias de química y física en el nivel medio del Colegio Nacional San Javier en el año 2020-2021, se desarrollo en el Colegio Nacional San Javier ubicada en la compañía de San Javier, en el municipio de San Ignacio en el departamento de Misiones en la República del Paraguay. La misma tuvo como objetivo general analizar las TIC utilizadas y las estrategias de enseñanza aplicadas para el desarrollo de las clases virtuales en la materia de química y física en el nivel medio del Colegio Nacional San Javier. La investigación fue de diseño no experimental, del tipo descriptivo de corte transversal. La población se conformó con 37 elementos en total, diferenciado por categorías de 2 docentes, 1 director y 34 estudiantes del nivel medio, las técnicas de recolección de datos fueron la encuesta y entrevista. Entre los hallazgos relevantes se encuentra que las aplicaciones de TIC más utilizadas en las clases virtuales son las más manipuladas en la actualidad, tales como el WhatsApp, Telegram, Meet, Zoom, correo electrónico y la plataforma del MEC.

Palabras clave: tic; estrategias de enseñanza; clase virtual; nivel medio 


\title{
The tic and teaching strategies applied in the development of virtual classes in the subjects of chemistry and physics at the secondary level of the colegio nacional San Javier in the year 2020-2021
}

\begin{abstract}
The research called ICT and the teaching strategies applied in the development of virtual classes in the subjects of chemistry and physics at the intermediate level of the San Javier National School in the year 2020-2021, was developed in the San Javier National School located in the company of San Javier, in the municipality of San Ignacio in the department of Misiones in the Republic of Paraguay. The same general objective was to analyze the ICT used and the teaching strategies applied for the development of virtual classes in the subject of chemistry and physics at the secondary level of the Colegio Nacional San Javier. The research was of a non-experimental design, of a descriptive cross-sectional type. The population was made up of 37 elements in total, differentiated by categories of 2 teachers, 1 director and 34 middle school students, the data collection techniques were the survey and interview, Among the relevant findings is that the most ICT applications used in virtual classes are currently the most manipulated, such as WhatsApp, Telegram, Meet, Zoom, email and the MEC platform.
\end{abstract}

Keywords: tic; teaching strategies; virtual class; medium level

Artículo recibido: 02 noviembre. 2021 Aceptado para publicación: 28 noviembre 2021 Correspondencia: luisaescurra@hotmail.com Conflictos de Interés: Ninguna que declarar 


\section{INTRODUCCIÓN}

La reforma educativa en el Paraguay se llevó a, dos años después de la caída de la dictadura. Su principal objetivo fue consolidar el régimen democrático, mejorar el desempeño escolar de jóvenes generaciones y desarrollar la competitividad económica nacional. La reforma educativa trajo consigo una variedad de cambio, en lo pedagógico, infraestructura, tecnológico y de cultural. Y el Colegio Nacional San Javier, no está excepta de este contexto, la institución educativa se encuentra en la compañía de San Javier en la circunscripción de la ciudad de San Ignacio. En el año 2020 con la pandemia del COVID-19, las clases presenciales pasaron obligatoriamente a ser virtuales, esta situación que afecto drásticamente a todo el mundo, no solo en el aspecto de la salud o en lo psicológico, también en lo económico y educativo. Con la pandemia fue necesario medidas drásticas en la forma de desarrollar las clases en todos los niveles del sistema educativo, y una de ellas fue la implementación de las clases virtuales mediante las TIC, en todos los niveles del sistema educativo del país, a pesar que desde un tiempo atrás la capacitación docente incluía la incorporación de las TIC en la enseñanza, esta vez las clases serian totalmente con el apoyo de las TIC, así se realizó desde 2020 hasta la fecha de la realización de la investigación. La nueva modalidad de clase, trajo consigo ventajas y desventajas, tanto del lado de los docentes y de los alumnos, la manera de desarrollar las clases fueron mediante las TIC, tales como las video conferencias, las redes sociales, las mensajerías instantánea, las plataformas educativas, esta nueva modalidad trajeron consigo un nueva mentalidad a la hora de enseñar y aprender, pero, esto también incluía un esfuerzo de parte de los docentes, estudiante y familiares del Colegio Nacional San Javier. La educación paraguaya es totalmente gratuita, pero, con el cambio de modalidad presencial a la virtual una de las desventajas en el país es que la mala señal de la internet, además la situación económica de las familias para acceder a una computadora, tablet o celulares de alta gama, para el desarrollo adecuado de las clases.

Por las razones citada se realizó la investigación denominada Las TIC y las estrategias de enseñanza aplicadas en el desarrollo de las clases virtuales en las materias de química y física en el nivel medio del Colegio Nacional San Javier en el año 2020-2021. La misma se realizó en la compañía de San Javier, dependiente del municipio de San Ignacio en el departamento de Misiones en la República del Paraguay, la temporalidad 
de la misma fue el año 2020-2021. El objetivo general fue analizar las TIC utilizadas y las estrategias de enseñanza aplicadas para el desarrollo de las clases virtuales en la materia de química y física en el nivel medio del Colegio Nacional San Javier.

\section{Y los objetivos específicos fueron:}

- Puntualizar las TIC que se usan en el desarrollo de las clases virtuales en las materias de química y física en el nivel medio del Colegio Nacional San Javier, ubicada en la compañía San Javier de la ciudad de San Ignacio Misiones.

- Cotejar las ventajas y desventajas en la utilización de las TIC en las clases virtuales en la materia de química y física en el nivel medio del Colegio Nacional San Javier, ubicada en la compañía San Javier de la ciudad de San Ignacio Misiones.

- Determinar las estrategias de enseñanza utilizadas en las clases virtuales en la materia de física y química en el nivel medio del Colegio Nacional San Javier, ubicada en la compañía San Javier de la ciudad de San Ignacio Misiones.

- Establecer la opinión de los estudiantes y los docentes en relación a las clases virtuales en la materia de química y física en el nivel medio del Colegio Nacional San Javier, ubicada en la compañía San Javier de la ciudad de San Ignacio Misiones.

\section{ESTRATEGIAS METODOLÓGICAS O MATERIALES Y MÉTODOS}

La investigación se desarrolló en el Colegio San Javier ubicada en la compañía denominado San Javier, en la circunscripción de la Ciudad de San Ignacio en el departamento de Misiones en la República del Paraguay. El diseño de investigación fue no experimental, puesto que no se manipulo de ninguna forma las variables en estudio Tecnología de la información y la comunicación y Estrategias de enseñanza; y el tipo de estudio fue descriptivo de corte transversal, porque solo se caracterizó las propiedades de las variables y se medio estas en un solo momento y no en forma secuencial en el tiempo. Los tipos de datos fueron de un enfoque mixto, es decir, los datos recabados tuvieron un carácter cualitativo y cuantitativo. Las fuentes primarias estuvieron conformadas por los docentes, directivos y estudiantes. La población estuvo conformada por 37 elementos, discriminado de la siguiente forma, un directivo, un docente de Física, un docente de química y 34 estudiantes del nivel medio del Colegio Nacional San Javier 
El marco operacional de la investigación fue como sigue:

\begin{tabular}{|c|c|c|c|}
\hline Variable & Definición conceptual & Definición operacional & Indicadores \\
\hline \multirow{6}{*}{ TIC } & \multirow{6}{*}{$\begin{array}{l}\text { Las TIC son el total de tecnologías } \\
\text { mediante las cuales se puede adquirir, } \\
\text { producir, almacenar, tratar, comunicar, } \\
\text { registrar y presentar informaciones, en } \\
\text { forma de voz, imágenes y datos } \\
\text { codificados en señales acústicas, ópticas } \\
\text { o electromagnéticas. (Manríquez, 2019, } \\
\text { p.35). }\end{array}$} & \multirow{6}{*}{$\begin{array}{l}\text { Con este constructor se } \\
\text { pretende determinar los } \\
\text { tipos, la interacción, } \\
\text { ventajas y desventajas del } \\
\text { uso del tic en las clases } \\
\text { virtuales en las materias } \\
\text { de física y química, } \\
\text { además de la opinión de } \\
\text { los estudiantes y docentes } \\
\text { en relación a la aplicación } \\
\text { de estas. }\end{array}$} & Tipos \\
\hline & & & Interacción \\
\hline & & & Ventajas \\
\hline & & & Desventaja \\
\hline & & & Limitaciones \\
\hline & & & Opinión \\
\hline \multirow{3}{*}{$\begin{array}{l}\text { Estrategias } \\
\text { de } \\
\text { enseñanza }\end{array}$} & $\begin{array}{l}\text { Estrategia de enseñanza: es el conjunto } \\
\text { de acciones y procedimientos, mediante } \\
\text { el empleo de métodos, técnicas, medios } \\
\text { y recursos que el docente emplea para }\end{array}$ & $\begin{array}{l}\text { Con la variable } \\
\text { pretende } \\
\text { estrablecer las } \\
\text { utrategias de enseñanza } \\
\text { utilizadas en las clases }\end{array}$ & Tipos \\
\hline & $\begin{array}{l}\text { planificar, aplicar y evaluar de forma } \\
\text { intencional, con el propósito de lograr } \\
\text { eficazmente el proceso educativo en una } \\
\text { situación de enseñanza-aprendizaje } \\
\text { específica, según sea el modelo }\end{array}$ & $\begin{array}{lcc}\text { virtuales de física } & \text { y } \\
\text { química, además } & \text { de } \\
\text { interacción y el rol del } \\
\text { docente durante las } \\
\text { clases. }\end{array}$ & Interacción \\
\hline & $\begin{array}{l}\text { pedagógico y/o andragógico por: } \\
\text { contenidos, objetivos y/o competencias } \\
\text { para las cuales las elabora y desarrolla, } \\
\text { (Guárate y Hernández, 2018, párr .1). }\end{array}$ & & $\begin{array}{l}\text { Rol del } \\
\text { docente }\end{array}$ \\
\hline
\end{tabular}

Las hipótesis de investigación expresa: las estrategias de enseñanza aplicadas en las clases virtuales en las materias de química y física en el nivel medio del Colegio San Javier son acordes a las necesidades específicas de la materia tales como plataforma, específicas del área y clases virtuales teóricas y demostrativas.

\section{MARCO TEÓRICO}

\subsection{TIC - Definición}

Para Sáez (2009), citado por Cordero, (2017), dice que: las tecnologías de la información comprenden el: "conjunto formado por las telecomunicaciones y la informática y todos sus antecedentes y consecuentes (microelectrónica, redes de ordenadores, ofimática, groupware, red Internet, tecnologías del multimedia, etc.), conjunto que, como infraestructura creciente en tamaño y capilaridad tendiente a la ubicuidad”. (p.30). 


\subsection{Características de las TIC.}

Por su parte Cordero, (2019, p4), dice que existe una infinidad de características las cuales definen a las TIC pero las que más resaltan en su trabajo de investigación son: Interactivas, Instantaneidad, Interconexión, Digitalización, Diversidad, Colaboración y la penetración en todos los sectores

\subsection{Ventajas y desventajas del uso de las TIC en educación.}

\section{Para el profesor.}

\section{Tabla A}

Ventajas y desventajas del uso de las TIC para el profesor

\begin{tabular}{|c|c|}
\hline & \\
\hline $\begin{array}{l}\text { profesor puede acceder a innumerables fuentes tanto de } \\
\text { nocimiento como metodológicas para el desarrollo de sus } \\
\text { tedras. Además, puede acceder a las publicaciones más } \\
\text { ciente sobre sus temas de investigación y publicación. }\end{array}$ & $\begin{array}{l}\text { e un } \\
\text { eccionamiento } \\
\text { tante de los profesores, } \\
\text { inversión de tiempo y } \\
\text { ro. }\end{array}$ \\
\hline cio & \multirow{2}{*}{$\begin{array}{l}\text { La existencia de mucha } \\
\text { información, exige que los } \\
\text { profesores dediquen } \\
\text { tiempo en analizar su } \\
\text { pertinencia y concluir que } \\
\text { no tienen errores. }\end{array}$} \\
\hline & \\
\hline & \multirow{2}{*}{$\begin{array}{l}\text { En ocasiones, el método } \\
\text { clásico exige menos } \\
\text { compromiso, tiempo y } \\
\text { esfuerzo }\end{array}$} \\
\hline & \\
\hline $\begin{array}{l}\text { Man } \\
\text { de } \\
\text { inve }\end{array}$ & \multirow{3}{*}{ 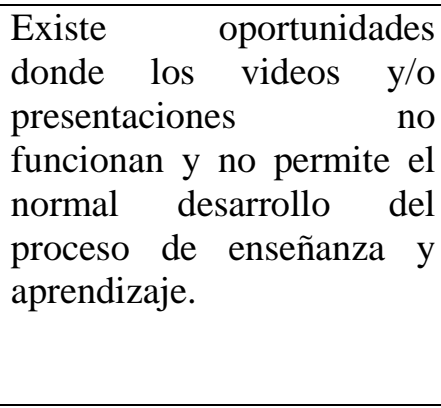 } \\
\hline & \\
\hline na de & \\
\hline Motiva a los pro & \multirow{2}{*}{$\begin{array}{l}\text { En ocasiones, los } \\
\text { profesores son muy } \\
\text { dependiente de r la } \\
\text { tecnología, y si algo falla } \\
\text { no pueden desarrollar la } \\
\text { clase. }\end{array}$} \\
\hline & \\
\hline
\end{tabular}

Fuente: (Díaz, 2017, pp.47- 48). 


\section{Para el estudiante.}

\section{Tabla B}

Ventajas y desventajas del uso de las TIC para el estudiante

\begin{tabular}{|c|c|}
\hline Ventajas & Desventajas \\
\hline $\begin{array}{l}\text { El acceder a múltiples recursos educativos para } \\
\text { estudiar y trabajar un determinado contenido. } \\
\text { Los estudiantes pueden aprender en menos tiempo, } \\
\text { en comparación con el aprendizaje tradicional. }\end{array}$ & $\begin{array}{l}\text { Como se tiene acceso a mucha } \\
\text { información, es muy fácil que los } \\
\text { estudiantes se distraigan en la } \\
\text { búsqueda de la información. Y en } \\
\text { algunas ocasiones, la información } \\
\text { obtenida es incorrecta. . }\end{array}$ \\
\hline $\begin{array}{l}\text { El trabajo es muy motivador, porque el trabajar con } \\
\text { tecnología atrae y llama la atención. }\end{array}$ & $\begin{array}{l}\text { Da lugar a que estudiantes se } \\
\begin{array}{l}\text { aprovechen } \\
\text { colaborativo, y no trabajen }\end{array}\end{array}$ \\
\hline $\begin{array}{l}\text { Hace que el proceso de enseñanza y aprendizaje se } \\
\text { desarrolle en función a las habilidades y cualidades } \\
\text { individuales, es decir, exista una personalización } \\
\text { del proceso enseñanza aprendizaje. } \\
\text { Existe una mayor cercanía con el profesor, porque } \\
\text { existen comunicación por correo electrónico, skype }\end{array}$ & $\begin{array}{l}\text { El acceso a muchas distracciones, es } \\
\text { conocidos por todos que cuando un } \\
\text { estudiante está trabajando, lo haga } \\
\text { estando conectado a diferentes redes } \\
\text { sociales. }\end{array}$ \\
\hline $\begin{array}{l}\text { El acceso a materiales en cualquier tiempo y lugar, } \\
\text { lo que permite una mayor flexibilidad de estudios. }\end{array}$ & $\begin{array}{l}\text { El acceder a mucha información, } \\
\text { puede llevar al plagio "cortar y pegar". }\end{array}$ \\
\hline $\begin{array}{l}\text { Motiva la iniciativa en la profundización de temas } \\
\text { trabajados o el estudio de otros nuevos, que sean de } \\
\text { interés para los estudiantes. }\end{array}$ & \\
\hline $\begin{array}{l}\text { Favorece el aprendizaje cooperativo entre } \\
\text { los estudiantes. }\end{array}$ & \\
\hline $\begin{array}{l}\text { Desarrolla la habilidad de búsqueda y selección de } \\
\text { información, de acuerdo a las necesidades y } \\
\text { requerimientos. }\end{array}$ & \\
\hline
\end{tabular}

Fuente: Fuente: (Díaz, 2017, p.48).

\subsection{Clases virtuales.}

Un aula virtual es un entorno digital en el que se puede llevar a cabo un proceso de intercambio de conocimientos que tiene por objetivo posibilitar un aprendizaje entre los usuarios que participan en el aula. En otras palabras, un aula virtual es un espacio dentro de una plataforma online en la que comparten contenidos profesores y alumnos, y en el que se atienden consultas, dudas y evaluaciones de los participantes. Como tal, el aula virtual no tiene límites físicos ni temporales. El alumno puede acceder a ella cuando quiera para tomar sus clases, sin tener que estar sujeto a horarios y a desplazamientos físicos. (Evol-Campus, 2016, párr. 4-5).

Los Elementos TIC utilizados en las clases virtuales son Celular, Tableta, Computadora de mesa, Notebook, Televisor, Radio, Internet móvil (con datos) y Internet banda ancha. Las Aplicaciones de mensajería instantánea más utilizadas en las clases virtuales son: 
WhatsApp, Telegram, Line, YouTube y Mensaje de texto normal. Las Herramientas colaborativas o aplicaciones de video conferencia y teletrabajos en las clases virtuales son: Zoom, Google Meet, Microsoft teams y Jitsi. Las aplicaciones de plataformas mas utilizadas son: Plataforma MEC, Correo electrónico, Classroom y Moodle. Las aplicaciones exclusivas para la enseñanza de la Química en forma virtual son; Tabla Periódica 2018, Glosario de química, Suite Química Gratuita y Química. Las aplicaciones móviles exclusivas para la enseñanza de la Física en forma virtual son: Física Básica - Resúmenes, Fórmulas y Figuras; Física - Tutoriales - Clases:

\subsection{Estrategias de enseñanza.}

Las estrategias de enseñanza son los diferentes procedimientos posibles para promover aprendizajes significativos (un nuevo aprendizaje para nuestros alumnos). La estrategia se va a dar según la realidad del alumno. Cada estrategia es dada por el profesor según su creatividad que permite conseguir un objetivo de aprendizaje. (Casimiro y Carhuavilca, 2010), citado por (Suni y Vásquez, 2018, p.28).

\section{RESULTADOS Y DISCUSIÓN}

\section{1. ¿Cuál de estos elementos Tic dispone para las clases virtuales?}

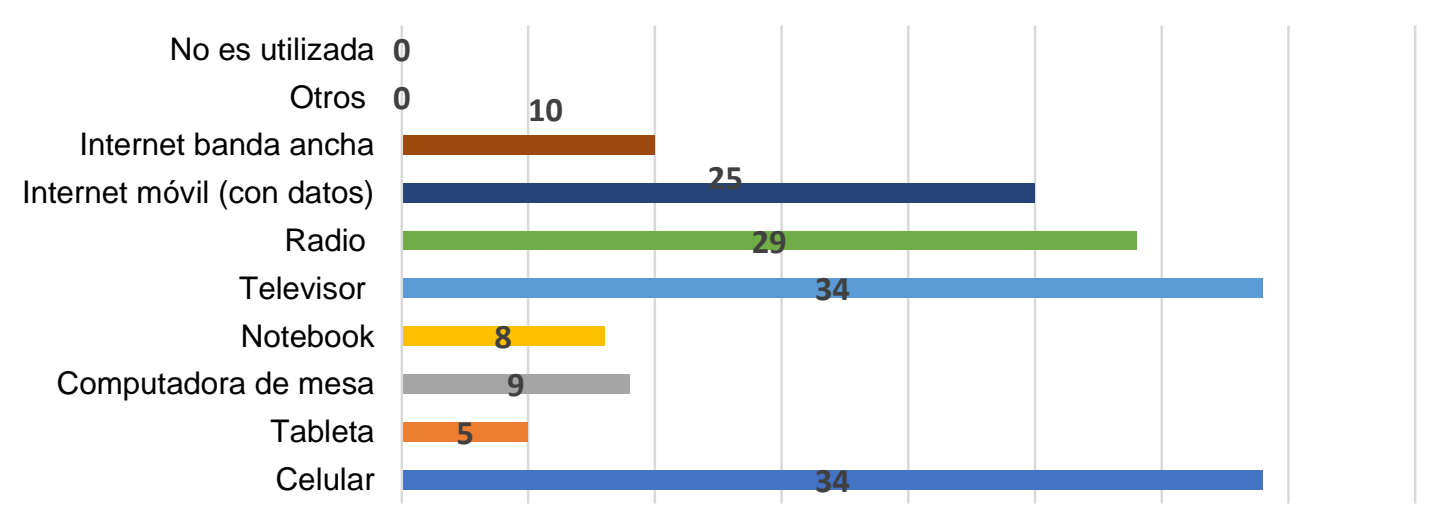

\section{Respuesta de los estudiantes}

En relación a la interrogante presentada 34 de los estudiantes comentaron que celular y televisor; 5 estudiantes indicaron que tableta; 9 estudiantes optaron que computadora de mesa; 8 estudiantes que notebook; 29 estudiantes que radio; 25 estudiantes que internet móvil y 10 de los mismos afirmaron que internet de banda ancha. 
4.2. ¿Cuál de estas aplicaciones de mensajería instantánea son más usadas en las clases virtuales en la materia de Física y Química?

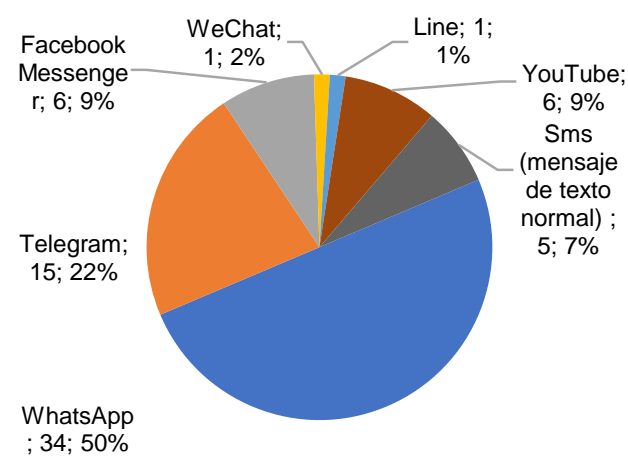

Respuesta para Física

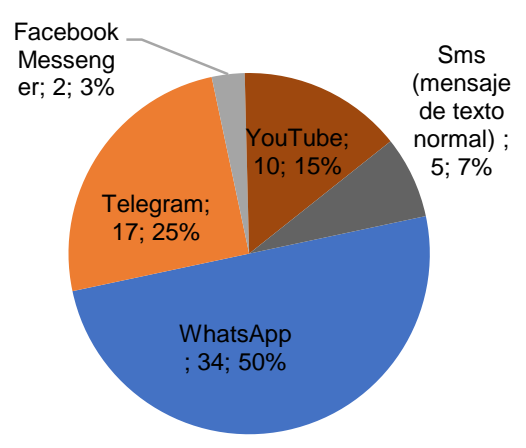

Respuesta para Química

Se evidencia que tanto para la materia de física y química las aplicaciones de mensajerías instantáneas más usadas son WhatsApp y Telegram, así lo expresan más del $70 \%$ de los estudiantes, seguida de YouTube y las mensajerías normales (SMS), estas aplicaciones son las más utilizadas también para la comunicación en la actualidad. Así también el docente de física, el docente de química y el director afirma que las aplicaciones de mensajerías instantáneas más usadas en las clases virtuales son el WhatsApp y Telegram, coincidiendo con las respuestas mayoritarias de los estudiantes.

4.3. ¿Cuál de estas aplicaciones de videoconferencia y teletrabajos son más usadas en las clases virtuales de Física y Química?

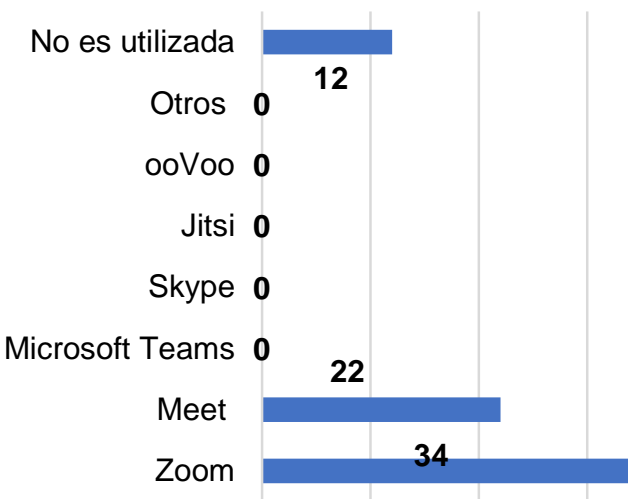

Respuesta para Física

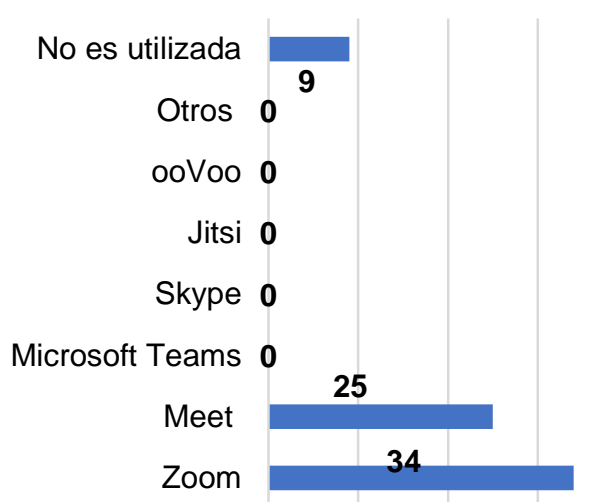

Respuesta para Química

En ambas materias la aplicación Zoom y Meet son las utilizadas según los estudiantes. Así mismo el docente de física, el docente de química y el director afirma que las mas utilizadas son el Zoom y el Meet, coincidiendo con las respuestas mayoritarias de los estudiantes 


\section{4. ¿Cuál de estas aplicaciones de plataforma y correo son más usadas en las clases virtuales de Física y Química?}

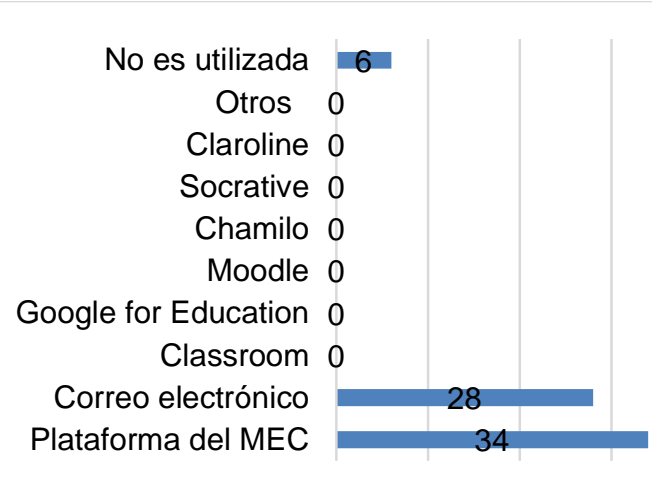

Respuesta para Física

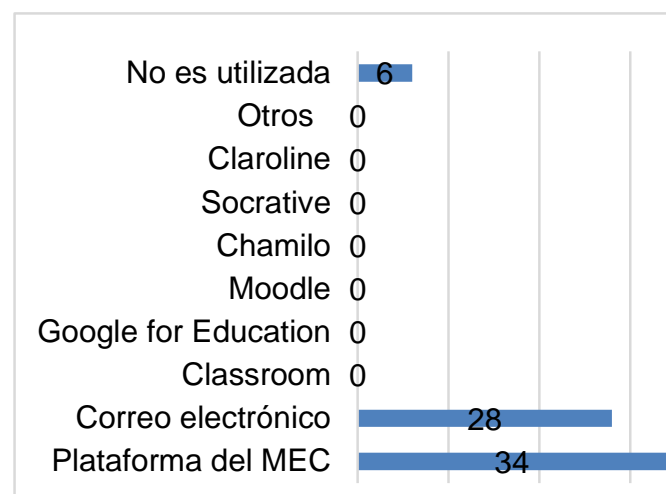

Respuesta para Química

En relación a la pregunta planteada a las aplicaciones de plataforma y correo más usadas en las clases virtuales de física y química son la plataforma del MEC y correo electrónico, pero existen estudiantes que dice que no se utiliza las plataformas. También el docente de física, el docente de química y el director afirma que las más utilizadas son el Zoom y el Meet, coincidiendo con las respuestas mayoritarias de los estudiantes.

\subsection{En tu opinión como estudiante, ¿Qué ventajas y desventajas tienen las TIC para los estudiantes en las clases virtuales?}

\begin{tabular}{|l|c|}
\hline \multicolumn{1}{|c|}{ Ventajas } & Cantidad \\
\hline $\begin{array}{l}\text { Acceso a varios recursos educativos para estudiar y trabajar en contenidos de la } \\
\text { materia de física y/o química. }\end{array}$ & 30 \\
\hline Puedo aprender en menos tiempo, en comparación con el aprendizaje en clase. & 6 \\
\hline $\begin{array}{l}\text { Las clases y trabajos prácticos son muy motivador, porque trabajar con tecnología } \\
\text { atrae y llama mi atención. }\end{array}$ & 13 \\
\hline $\begin{array}{l}\text { El proceso de enseñanza y aprendizaje se desarrolla en función a mis habilidades y } \\
\text { cualidades, es decir, la enseñanza aprendiza es personalizado. }\end{array}$ & 3 \\
\hline $\begin{array}{l}\text { Existe una mayor cercanía con mi profesor, porque puedo comunicarme por correo } \\
\text { electrónico, mensajes, videollamadas, etc. }\end{array}$ & 8 \\
\hline $\begin{array}{l}\text { Puedo acceder a materiales en cualquier tiempo y lugar, lo que permite una mayor } \\
\text { flexibilidad en mi estudio. }\end{array}$ & 18 \\
\hline $\begin{array}{l}\text { Favorece el aprendizaje cooperativo entre los estudiantes, es decir, puedo trabajar en } \\
\text { grupo de menor forma. }\end{array}$ & 10 \\
\hline $\begin{array}{l}\text { Se desarrolla mis habilidades de búsqueda y selección de información, de acuerdo a } \\
\text { las necesidades y requerimientos de cada materia }\end{array}$ & 5 \\
\hline $\begin{array}{l}\text { Los recursos son ricos y diferentes como pueden ser los vídeos, webs, gráficos y } \\
\text { juegos, resultan mejores que las materias más tradicionales. }\end{array}$ & 4 \\
\hline $\begin{array}{l}\text { Seguimiento, permite establecer tiempos de entrega para que el participante pueda } \\
\text { organizar las tareas. }\end{array}$ & 3 \\
\hline $\begin{array}{l}\text { Interactividad, permite que los participantes sean más activos y constructores de su } \\
\text { propio aprendizaje. }\end{array}$ & 2 \\
\hline Total & 102 \\
\hline
\end{tabular}




\begin{tabular}{|l|c|}
\hline \multicolumn{1}{|c|}{ Desventaja } & Cantidad \\
\hline $\begin{array}{l}\text { Como se tiene acceso a mucha información, es muy fácil que me distraiga en la } \\
\text { búsqueda de la información. }\end{array}$ & 24 \\
\hline Algunas informaciones obtenidas son incorrecta o no veraces. & 12 \\
\hline $\begin{array}{l}\text { Da lugar a que algunos estudiantes se aprovechen del aprendizaje colaborativo } \\
\text { (grupal), y no trabaje todo el grupo solo algunos. }\end{array}$ & 34 \\
\hline $\begin{array}{l}\text { El acceso a muchas distracciones, puede que cuando se está estudiando, lo haga } \\
\text { estando conectado a diferentes redes sociales. }\end{array}$ & 18 \\
\hline El acceder a mucha información, puede llevar al plagio “cortar y pegar”. & 14 \\
\hline Total & 102 \\
\hline
\end{tabular}

\section{Respuesta de los estudiantes}

Según los estudiantes la ventaja que se encuentra entre la más elegidas es que se puede tener acceso a varios recursos educativos para estudiar y trabajar en contenidos de la materia de física y/o química, la segunda opción más relevante fue que puede tener acceso a materiales en cualquier tiempo y lugar, por tercera opción más escogida que las clases y trabajos prácticos son muy motivador. Según el docente de física y el de química las ventajas que tienen las TIC para los estudiantes en las clases virtuales son que la enseñanza aprendizaje es personalizado, favorece el aprendizaje cooperativo entre los estudiantes, se desarrolla mis habilidades de búsqueda y selección de información, de acuerdo a las necesidades y requerimiento de cada materia. Para los estudiantes las desventajas son que otros estudiantes se aprovechen del aprendizaje colaborativo (grupal), y no trabaje todo el grupo solo algunos, como segunda opción esta que es muy fácil que se distraiga en la búsqueda de la información por las múltiples conexiones que se puede realizar en las redes sociales. Lo mismo expresaron los docentes en ambas materias y el director, agregando la situación que en los trabajos se puede realizar plagios, es decir copiar y pegar.

4.6. ¿Cuál de esta estrategia de enseñanza utiliza tu profesor de física en las clases virtuales?
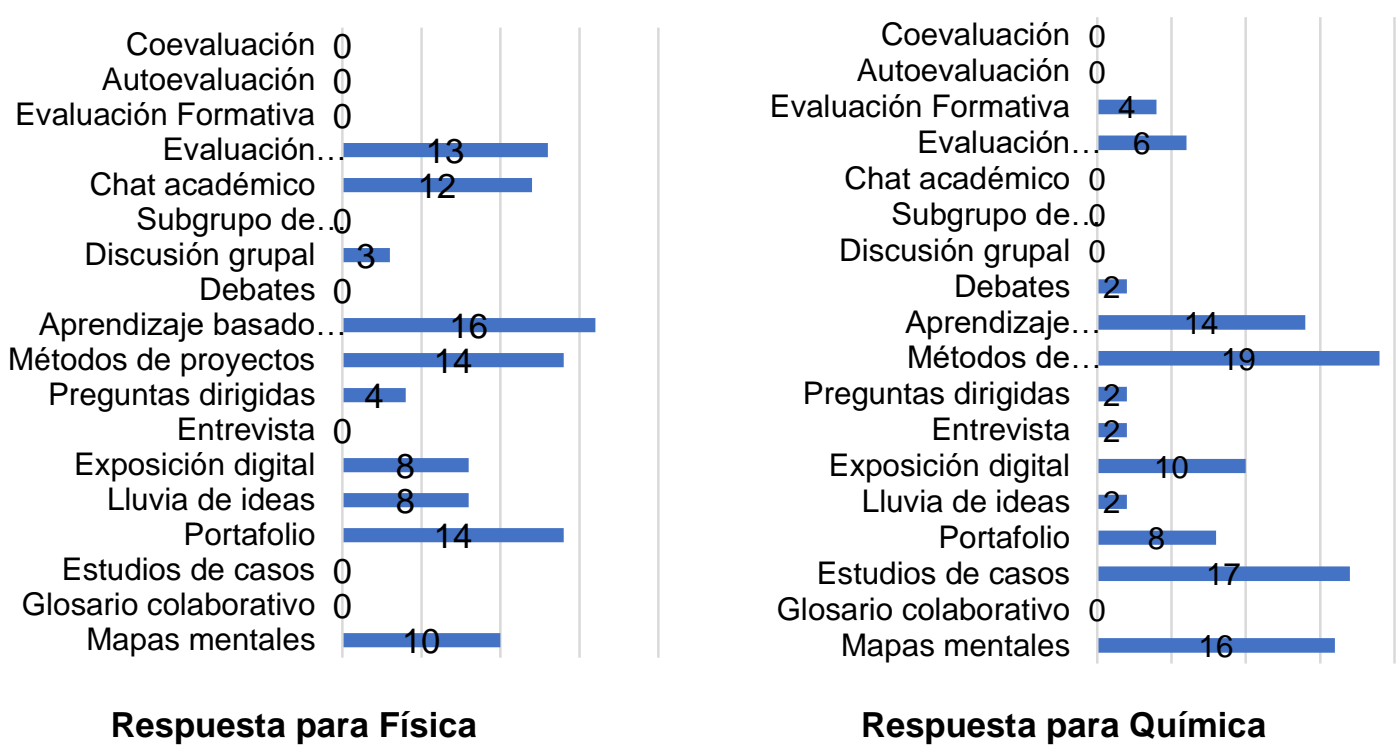

Respuesta para Química 
En la materia de física las cuatro principales estrategias de enseñanza más utilizadas por el docente según los estudiantes son aprendizaje basado en problemas, métodos de proyectos, portafolio y chat académico. Y en las clases de química las más usadas son método de proyecto, estudio de casos, mapas mentales y aprendizaje basados en problemas. Las estrategias en común son el método de proyecto y aprendizaje basado en problemas. Según el docente de física las estrategias que utiliza en las clases virtuales son portafolio, lluvia de ideas y evaluación formativa, así también el docente de química expreso que usa el portafolio, lluvia de ideas y evaluación formativa.

\subsection{En tu opinión ¿Qué rol desempeñan tu profesor en las clases virtuales de física y química?}

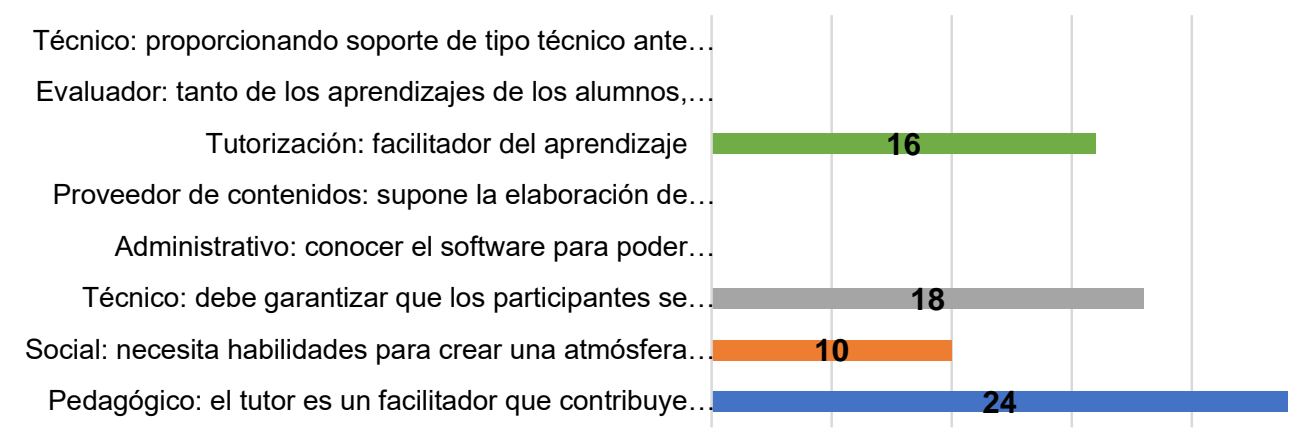

Para la mayoría de los estudiantes el rol del docente en las clases virtuales es de pedagógico, la segunda opción más elegida fue la de técnico, le sigue el rol de tutorización y por último el rol social. Este primer rol de pedagogo es también la opción que el docente de física, química, y director consideran que realizan los docentes en las clases virtuales.

\section{CONCLUSIÓN O CONSIDERACIONES FINALES}

Teniendo en cuenta la literatura recabada, los resultados obtenidos mediante los instrumentos de recolección de datos y atendiendo los objetivos específicos se concluye: En cuanto a las TIC que se usan en el desarrollo de las clases virtuales en las materias de química y física en el nivel medio del Colegio Nacional San Javier, por orden de cantidad los estudiantes disponen de elementos Tic tales como celular, televisor, radio, internet con datos móviles, internet banda ancha, computadora de mesa y por último tableta. Ahora bien, el docente de química y física, así como el director coinciden que los estudiantes disponen de celular en primer lugar, seguido de computadora de mesa y 
notebook, televisor, radio, internet con datos móviles y por último internet de banda ancha.

En relación a las aplicaciones más utilizadas en las clases virtuales en la materia de química y física en el nivel medio del Colegio Nacional San Javier, las dos principales mensajerías instantáneas más utilizadas en ambas materias son, en primer lugar, WhatsApp y en segundo lugar Telegram, para las video conferencias y teletrabajo, Zoom y Meet, en cuanto a las aplicaciones de plataforma y correos los estudiantes respondieron que el más utilizado es la plataforma del MEC seguido del correo electrónico. También el docente de química y física, así como el director coinciden con los estudiantes que estas son las aplicaciones más utilizadas en las clases virtuales de física y química, ahora bien, en cuanto a las aplicaciones específicas para la materia de Química según los estudiantes son Tabla periódica 2018 y Glosario de química, para la materia de física son Física Básica - Resúmenes, Fórmulas y Figuras, el docente de física coincide con la primera aplicación especifica que expresaron los estudiantes, pero para la segunda según el docente es Física tutoriales y clases. La aplicación especifica más usada en química según el docente es la Tabla periódica 2018, en coincidencia con los estudiantes, pero discrepa en la segunda que es Química, en cambio el directo expreso que en las dos materias no se utiliza ninguna aplicación especifica, en contraposición a lo expresado por los estudiantes y docentes.

En relación a las ventajas y desventajas en la utilización de las TIC en las clases virtuales en la materia de química y física en el nivel medio del Colegio Nacional San Javier, según los estudiantes las tres principales ventajas de las TIC en las clases presenciales son, acceso a varios recursos educativos para estudiar y trabajar en contenidos de la materia de física y/o química, acceder a materiales en cualquier tiempo y lugar, que las clases y trabajos prácticos son muy motivador. Para el docente de Física las ventajas en utilización de las TIC en las clases virtuales son que la enseñanza aprendizaje es personalizado, favorece el aprendizaje cooperativo entre los estudiantes, y se desarrolla mis habilidades de búsqueda y selección de información, de acuerdo a las necesidades y requerimientos. Las ventajas para el docente de Química fue que, el acceso a varios recursos educativos para estudiar, puedo acceder a materiales en cualquier tiempo y lugar, favorece el aprendizaje cooperativo entre los estudiantes. Entre las desventajas citadas por los estudiantes en cuanto a la utilización de las TIC en 
las clases virtuales, en primer lugar, se encuentra a que las TIC da lugar a que algunos estudiantes se aprovechen del aprendizaje colaborativo (grupal), y no trabaje todo el grupo solo algunos, es muy fácil que me distraiga en la búsqueda de la información y que el acceso a muchas distracciones, puede que cuando se está estudiando, lo haga estando conectado a diferentes redes sociales. El docente de Física y de Química coinciden con los estudiantes en relación las desventajas, pero agrega que el acceder a mucha información, puede llevar al plagio "cortar y pegar". Ahora bien, las ventajas de las TIC específicamente en el aprendizaje de los estudiantes, según la mayoría de ellos es que permite que los estudiantes y profesores tengan acceso rápido a la información e intercambio de la misma, le sigue que facilita el aprendizaje en grupo y se desarrollan las habilidades sociales, mediante el intercambio de información. Y la tercera opción fue permite una mayor comunicación entre el profesor y el estudiante, que va más allá de la sala de clases, ya se puede comunicar por correo electrónico, plataformas. En relación al punto el docente de física expreso que las TIC elimina las barreras de tiempo y espacio en el desarrollo de las de enseñanza y aprendizaje; permite una mayor comunicación entre el profesor ya se puede comunicar por correo electrónico, plataformas o mensajerías y se facilita el aprendizaje en grupo y se desarrollan las habilidades sociales, mediante el intercambio de información. Y el docente de química que las TIC permite una enseñanza personalizada, porque existe la posibilidad de adaptar la información y las actividades a las características de los estudiantes, permite que los estudiantes y profesores tengan acceso rápido a la información e intercambio de la misma y permiten una alfabetización constante, porque exige que los profesores y alumnos estén en una búsqueda constante de contenidos, también el director dice que las TIC elimina las barreras de tiempo y espacio en el desarrollo de las de enseñanza y aprendizaje, permite que los estudiantes y profesores tengan acceso rápido a la información e intercambio de la misma y ofrece la posibilidad de desarrollar habilidades de expresión escrita, gráfica y audiovisual. Y entre las desventajas de las TIC en relación al aprendizaje los estudiantes expresaron que, si no se trabaja conscientemente, se corre el riesgo de que los aprendizajes sean incompletos, que el costo de la tecnología es elevado, por lo que es necesario poseer o tener a disposición recursos para acceder a ella y por último que, en ocasiones, es necesario la actualización de equipos y software para poder desarrollar las tareas. Las respuestas dadas por los estudiantes en las 
desventajas coinciden con el docente de físca, química y el director en los puntos que, si no se trabaja conscientemente, se corre el riesgo de que los aprendizajes sean incompletos y da lugar a que se realicen plagios, además que el costo de la tecnología es elevado, por lo que es necesario poseer o tener a disposición recursos para acceder a ella y que puede existir una excesiva dependencia entre los participantes del proceso de enseñanza y aprendizaje y da lugar a que se realicen plagios. Las estrategias de enseñanza utilizadas en las clases virtuales en la materia de y física en el nivel medio del Colegio Nacional San Javier, ubicada en la compañía San Javier de la ciudad de San Ignacio Misiones, por orden de prioridad, las cinco más utilizadas según los estudiantes son el aprendizaje basado en problemas, el portafolio, el método de proyecto, la evaluación formativa y el chat académico. En cambio, en las clases de química las estrategias más relevantes según los estudiantes son el método de proyectos, estudio de casos, mapas mentales, aprendizaje vasados en problemas y exposición digital. Entre las estrategias de enseñanza utilizadas en las clases de física y química existen coincidencia en Aprendizaje basado en problemas y Métodos de proyectos. En cambio, el docente de física alega que utiliza más las estrategias de enseñanza como el portafolio, lluvia de ideas y evaluación formativa; y el docente de química las estrategias de portafolio, lluvia de ideas y evaluación formativa. Así también el director expresó que las estrategias más utilizadas en estas materias son mapas mentales, portafolio y aprendizaje basado en problemas. Entre las estrategias de enseñanza citas por los docentes y el director la coincidencia parcial son las estrategias de aprendizaje basado en problemas y portafolio. Entre todos los actores la estrategia más utilizada en las clases virtuales es el aprendizaje basado en problemas.

En cuanto a la opinión de los estudiantes en relación a las clases virtuales en la materia de química y física en el nivel medio del Colegio Nacional San Javier, los estudiantes consideran que el docente cumple el rol de pedagógico la opción más elegida, le sigue el rol de técnico y tutorización. El rol pedagógico es la coincidencia entre lo expresado por los estudiantes, director y docentes. Con respecto a la interacción entre estudiante/contenido, la interacción Estudiante/profesor, la interacción estudiante/estudiante y la interacción tiempo/aprendizaje la mayoría de los estudiantes expresaron que es buena, así también la interacción/tiempo de duración de las clases virtuales en las materias de física y química. El docente de química coincide con los 
estudiantes que en todas las interacciones la calificación es buena, en cambio el director expresó que la calificación en todas las interacciones muy buena, Solo el docente de física expresó que la interacción estudiante/profesor y el tiempo/aprendizaje es muy buen, además que la interacción estudiante/contenido es excelente y la interacción estudiante/estudiante es buena.

Teniendo en cuenta lo expresado y el objetivo general de la investigación, se resume que las TIC más utilizadas para las clases virtuales de física y química en el Colegio Nacional San Javier son las masajearías instantáneas WhatsApp y Telegram, entre las aplicaciones de video conferencias y teletrabajo están el Zoom y Meet, en cuanto a las aplicaciones de plataforma y correos el más utilizado es la plataforma del MEC. Además, las TIC que dispone los estudiantes está el celular, la conexión a internet por datos móviles y alguna computadoras y notebook. De las estrategias lo llamativo es que son las mismas que las clases presenciales, exceptuando el chat online, puesto que las estrategias de enseñanza lluvia de ideas, la evaluación formativa, el portafolio y el aprendizaje basado en problemas se aplican también a las clases presenciales, todo esto se lleva a cabo con una interacción con una calificación media de buena (tres) de la escala de cinco. Además, la limitación principal es el costo de conexión y los equipos TIC (informáticos), también cabe destacar que no se aprovecha del todo las aplicaciones específicas para cada materia, en la actualidad existen aplicaciones diversas para demostrar la teoría mediante las aplicaciones. De esta forma se comprueba en forma parcial la hipótesis de investigación, puesto que se utilizan TIC apropiadas, pero es necesario mayor interacción y aplicaciones específicas para cada metería para las demostraciones prácticas.

\section{LISTA DE REFERENCIAS}

Bembibre, C., (2011). Definición de Mensaje de Texto. Recuperado de https://www.definicionabc.com/comunicacion/mensaje-de-texto.php

Caballero, C., (2016). Los límites de la educación virtual. Recuperado de https://www.portafolio.co/opinion/cesar-caballero-reinoso/los-limites-de-laeducacion-virtual-cesar-caballero-540465

Cáceres, C., (2018). Microsoft TEAMS. Recuperado de https://www.ibermatica365.com/todo-lo-que-necesitas-saber-sobre-microsoftteams/ 
Catalaño, C., (2017). Definición de Jitsi. Recuperado de https://bit4learn.com/es/plataformas-para-webinars/jitsi-meet/

Chávez, A., (2016). ¿En qué consiste claroline?. Recuperado de http://plataformaeducativaclaroline.blogspot.com

Cuesta, H., (2010). TICS - Aulas Virtuales. Recuperado de https://es.slideshare.net/heivercuesta/tics-aulas-virtuales

Díaz, D., (2017). TIC en educación superior: ventajas y desventajas. Recuperado de https://dialnet.unirioja.es/servlet/articulo?codigo=5072156

Elantia. (2020). Google Meet, sus características y utilidad en educación. Recuperado de https://elantia.es/google-meet-sus-caracteristicas-y-su-uso-en-educacion/

Evol-Campus. (2016). ¿Qué es un aula virtual y para qué se puede utilizar?. Recuperado de https://www.evolmind.com/latam/blog/que-es-un-aula-virtual-ypara-que-se-puede-utilizar

Fernández, H., (2020). Qué es zoom y cómo funciona. Recuperado de https://economiatic.com/que-es-zoom-como-funciona/

Gamarra, C., (2021). Definición de televisión. Recuperado de https://conceptodefinicion.de/television/

Guárante, A., y Hernández, C., (2018). Que son las estrategias de enseñanza. Recuperado de https://www.magisterio.com.co/articulo/que-son-las-estrategiasde-

ensenanza\#: :text=Es\%20el\%20conjunto\%20de\%20acciones,específica\%2C\%2 0según\%20sea\%20el\%20modelo

LucusHost. (2020). ¿Qué es Moodle y para qué sirve?. Recuperado de https://www.lucushost.com/blog/que-es-moodle/

Maldonado, D., (2018). Claroline, una plataforma educativa virtual. Recuperado de https://empresayeconomia.republica.com/recursos-humanos/claroline-unaplataforma-educativa-virtual.html

Manríquez, A., (2019). El uso de TIC en la comunicación con la ciudadanía: diagnóstico de portales web de gobiernos locales en México. Universidad Complutense de Madrid. Facultad de Ciencias de la Información. Recuperado de https://eprints.ucm.es/id/eprint/50692/1/T40750.pdf 
Las TIC y las estrategias de...

Merayo, P., (2020). ¿Qué es Moodle?. Recuperado de https://www.maximaformacion.es/e-learn/que-es-moodle-y-para-que-sirve/

Pérez, J., y Gardey, A., (2013). Definición de plataforma virtual. Recuperado de https://definicion.de/plataforma-virtual/

Pérez, J., y Gardey, A., (2015). Definición de tabla periódica. Recuperado de https://definicion.de/tabla-periodica/

Pérez, J., y Merino, M., (2010). Definición de netbook. Recuperado de https://definicion.de/netbook/

Pérez, M., (2021). Correo Electrónico. Recuperado de https://conceptodefinicion.de/correo-electronico/

Pradillo, B., (2019). Apps para aprender química. Recuperado de https://www.orbitalesmoleculares.com/apps-para-aprender-quimica/ 